\title{
EMT reversal in human cancer cells after IR knockdown in hyperinsulinemic mice
}

\author{
Zara Zelenko', Emily Jane Gallagher1, Irini Markella Antoniou1, Deepali Sachdev², \\ Anupma Nayak ${ }^{3}$, Douglas Yee ${ }^{2}$ and Derek LeRoith'
}

${ }^{1}$ Division of Endocrinology, Diabetes and Bone Diseases, Icahn School of Medicine at Mount Sinai, New York, New York, USA

2Department of Medicine and Masonic Cancer Center, University of Minnesota, Minneapolis, Minnesota, USA ${ }^{3}$ Department of Pathology and Laboratory Medicine, The Mount Sinai Hospital and Icahn School of Medicine at Mount Sinai, New York, New York, USA
Correspondence should be addressed to D LeRoith Email Derek.LeRoith@mssm.edu

\begin{abstract}
Type 2 diabetes (T2D) is associated with increased cancer risk and cancer-related mortality. Data herein show that we generated an immunodeficient hyperinsulinemic mouse by crossing the Rag1-l- mice, which have no mature B or T lymphocytes, with the MKR mouse model of T2D to generate the Rag1-/- (Rag/WT) and Rag $1^{-/-/ M_{K}+/+}$ (Rag/MKR) mice. The female Rag/MKR mice are insulin resistant and have significantly higher nonfasting plasma insulin levels compared with the Rag/WT controls. Therefore, we used these Rag/MKR mice to investigate the role of endogenous hyperinsulinemia on human cancer progression. In this study, we show that hyperinsulinemia in the Rag/MKR mice increases the expression of mesenchymal transcription factors, TWIST1 and $Z E B 1$, and increases the expression of the angiogenesis marker, vascular endothelial growth factor A (VEGFA). We also show that silencing the insulin receptor (IR) in the human LCC6 cancer cells leads to decreased tumor growth and metastases, suppression of mesenchymal markers vimentin, SLUG, TWIST1 and ZEB1, suppression of angiogenesis markers, VEGFA and VEGFD, and re-expression of the epithelial marker, E-cadherin. The data in this paper demonstrate that IR knockdown in primary tumors partially reverses the growth-promoting effects of hyperinsulinemia as well as highlighting the importance of the insulin receptor signaling pathway in cancer progression, and more specifically in epithelial-mesenchymal transition.
\end{abstract}
Key Words
- type 2 diabetes
- cancer
- hyperinsulinemia
- insulin receptor
- epithelial-mesenchymal transition

Endocrine-Related Cancer (2016) 23, 747-758

\section{Introduction}

Both obesity and type 2 diabetes (T2D) are associated with increased cancer risk and cancer-related mortality (Haslam \& James 2005, Onitilo et al. 2012, Zhang et al. 2012). Obesity and T2D may contribute to cancer development and progression through several potential mechanisms. Both metabolic disorders are associated with systemic insulin resistance, hyperinsulinemia and increased growth factors, such as insulin-like growth factor 1 (IGF1), and altered adipokines, including adiponectin and leptin, which are reported to promote tumor growth and progression (Hursting et al. 2012). Furthermore, systemic and local adipose tissue inflammation with macrophage inflammation is prevalent in obesity and has been shown to contribute 
to tumor progression (Howe et al. 2013, Morris et al. 2013). Dyslipidemia is also a clinical characteristic of obesity and T2D and has been shown to contribute to breast cancer progression in mouse models (Alikhani et al. 2013).

Of all the possible factors involved in cancer progression and mortality, hyperinsulinemia is apparently the most egregious. A strong association between circulating insulin and lower progression-free survival has been shown in multiple studies (Goodwin et al. 2002, Eliassen et al. 2007).

To study whether endogenous hyperinsulinemia promotes the growth and progression of human metastatic cancer, we generated an immunedeficient MKR mouse by crossing the recombinationactivating gene 1 deleted (Rag1-l-) mouse with the hyperinsulinemic MKR mouse. The Rag $1^{-/-}$has been characterized as $\mathrm{B}$ and $\mathrm{T}$ cell deficient, whereas innate immunity is still functional (Mombaerts et al. 1992). The lack of $B$ and T cells allowed us to introduce human cancer cells to test the effect of hyperinsulinemia on tumor growth and metastases. In this study, we characterized the novel Rag1-/-/MKR+/+ (Rag/MKR) mice, an immunodeficient T2D mouse model. We induced human metastatic cancer cells (LCC6, a metastatic variant of the MDA-MB-435 cells) in the Rag/MKR and Rag/WT control mice. The origin of the MDA-MB-435 cells is controversial and it has been noted that the MDA-MB-435 cells display characteristics of both breast cancer and melanoma (Chambers 2009). Although the origin is controversial, we used the variant of the MDA-MB-435 cells because they are an established metastatic human cancer cell line, with expression of both epithelial and melanocyte markers (Chambers 2009). Our results determined that endogenous hyperinsulinemia profoundly increased the growth of human LCC6 cancers and that insulin receptor knockdown led to significantly decreased tumor growth in control and hyperinsulinemic mice. Furthermore, the knockdown of the insulin receptor led to changes in tumor signaling and uncovered the downregulation of mesenchymal markers and re-expression of epithelial marker, E-cadherin. To our knowledge, this is the first study to show that hyperinsulinemia promotes the increase in epithelial-mesenchymal transition (EMT) gene expression in human tumor xenografts. Moreover, this is the first study to show that insulin receptor knockdown leads to a reversal of the epithelial-mesenchymal phenotype in any human tumor xenografts.

\section{Methods}

\section{Animal models}

The hyperinsulinemic female MKR mouse has been well characterized and described in previous publications (Fernandez et al. 2001, Novosyadlyy et al. 2010, Ferguson et al. 2012). Briefly, the male mouse develops T2D with insulin resistance, hyperinsulinemia, hyperglycemia and hyperlipidemia between 7 and 8 weeks of postnatal life, and is nonobese since the model was developed
A
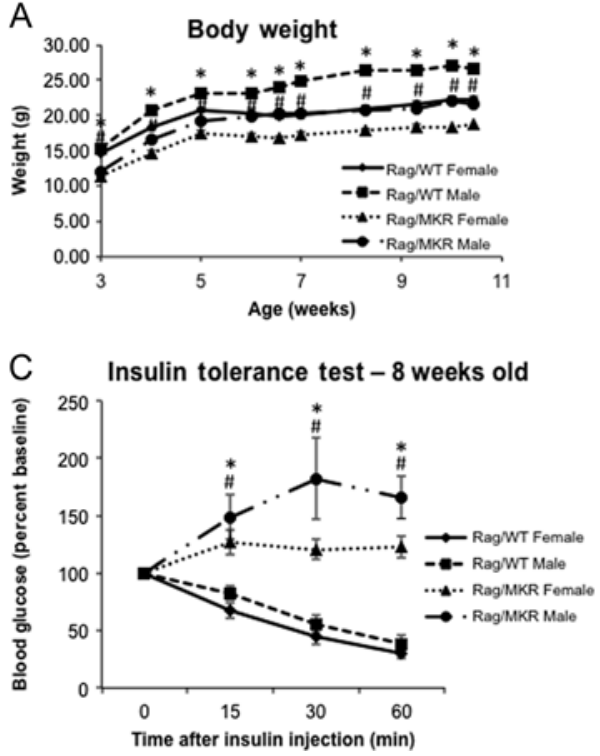

B

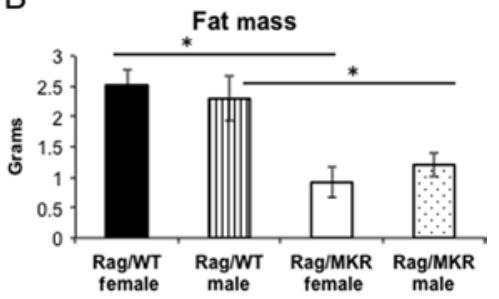

D

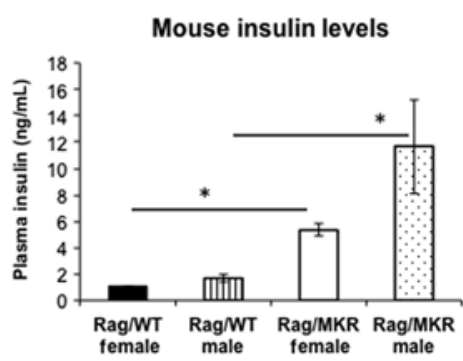

Figure 1

Characterization of an immunodeficient hyperinsulinemic mouse model. (A) Body weights of male and female mice from Rag/WT and Rag/MKR mice, measured weekly from 3 to 10 weeks of age. ${ }^{*} P<0.05$ between Rag/WT male and Rag/MKR male mice. $\# P<0.05$ between Rag/NT female and Rag/MKR female mice. $n=5$ mice per group, error bars represent S.E.M. (B) MRI results showing whole body fat in Rag/WT and Rag/MKR mice. $n=5$ mice per group, error bars represent S.E.M. (C) Insulin tolerance test (ITT) performed on fasted 8-week male and female Rag/NT and Rag/MKR mice. Blood glucose levels were measured at time 0 and 15, 30 and $60 \mathrm{~min}$ post insulin injection. Mice were injected with $0.75 \mathrm{U} / \mathrm{kg}$ insulin. ${ }^{*} P<0.05$ between Rag $/$ WT male and Rag/MKR male mice. ${ }^{*} P<0.05$ between Rag/NT female and Rag/MKR female mice. $n=5$ mice per group, error bars represent s.E.M. (D) Plasma insulin levels of nonfasted male and female Rag/WT and Rag/MKR mice. ${ }^{*} P<0.05$ between groups as indicated. Graphs represent the mean of each group ( $n=5$ mice per group), error bars are s.E.M. 
by genetically engineered severe muscle insulin resistance. The female mouse, on the other hand, is insulin resistant and hyperinsulinemic, but has normal blood glucose and lipid levels, and thus is a model of endogenous hyperinsulinemia in the absence of obesity and overt diabetes.

The Rag1-/- mice on a Friend Virus B (FVB) background, a kind gift of Dr Lisa Coussens (Oregon Health and Science University, Portland, OR, USA), were crossed with the $\mathrm{MKR}^{+/+}$mouse to generate homozygous Rag1-/-/MKR ${ }^{+/+}$(Rag/MKR) mice. All mice used in these studies were backcrossed to an FVB/National Institutes of Health (FVB/n) background. Tumor studies were commenced in female mice between 8 and 10 weeks of age.

\section{Animal studies}

All mouse procedures were in compliance with the current standards specified in the Guide of the Care and Use of Laboratory Animals provided by the Association for Assessment and Accreditation of Laboratory Animal Care (AAALAC) and approved by Mount Sinai Institutional Animal Care and Use Committee. The mice were housed 4-5 per cage, kept on a $12 \mathrm{~h}$ light: $12 \mathrm{~h}$ darkness cycle and fed a regular chow diet (PicoLab 5053, Brentwood, MO, USA). At 8 weeks of age, $5 \times 10^{6}$ LCC 6 control or LCC6 insulin receptor knockdown cells resuspended in $100 \mu \mathrm{L}$ of DMEM were injected into the 4th mammary fat pad of Rag/WT and Rag/MKR mice, 8-10 mice per group. For all studies, body weight was monitored weekly, and tumor size was measured twice weekly with calipers. The formula used for the calculating tumor volume was: volume $=4 / 3 \times \mathrm{pi} \times($ length $/ 2 \times$ width $/ 2 \times$ depth $/ 2)$. In order to analyze the pulmonary metastasis, postmortem lungs were inflated with 10\% formalin, paraffin embedded, sectioned and hematoxylin and eosin stained. Micrometastasis was examined under the microscope by a pathologist (Dr A Nayak).

\section{Real-time qPCR}

RNA was extracted from cells using the RNeasy Mini Kit (Qiagen) according to the manufacturer's instructions. One microgram of RNA was reverse-transcribed to cDNA using oligo (dT) primers with a RT-PCR kit according to the manufacturer's instructions (Invitrogen). Following reverse transcription, cDNA was subjected to real-time PCR using the QuantiTect SYBR green PCR kit (Qiagen) in ABI PRISM 7900HT sequence detection systems (Applied Biosystems).
Insulin Receptor Primers: 5'TACCCCGGAGAGGTGTGTC; 3'CCCGGAAGAGCAGCAAGTAA. ZEB1 primers: 5'CAGA GGATGACCTGCCAACA; 3'GCCCTTCCTTTCCTGTGTCAT. SLUG primers: 5'TTTCTGGGCTGGCCAAACAT; 3'TTCTC CCCCGTGTGAGTTCTA. TWIST1 primers: 5'GCCGGA GACCTAGATGTCATT; 3'CCCACGCCCTGTTTCTTTGA. VEGFA primers: 5'AGGCCAGCACATAGGAGAGA; 3'TTTCTTGCGCTTTCGTTTTT. VEGFD primers: 5'CCATC CAGATCCCTGAAGAA; 3'TTCCAGCAAGTGGATTTTCC. Ribosomal protein L19 (RPL19) housekeeping gene primers: 5'AGCTCTTTCCTTTCGCTGCT; 3'GATCTGCTG ACGGGAGTTGG.

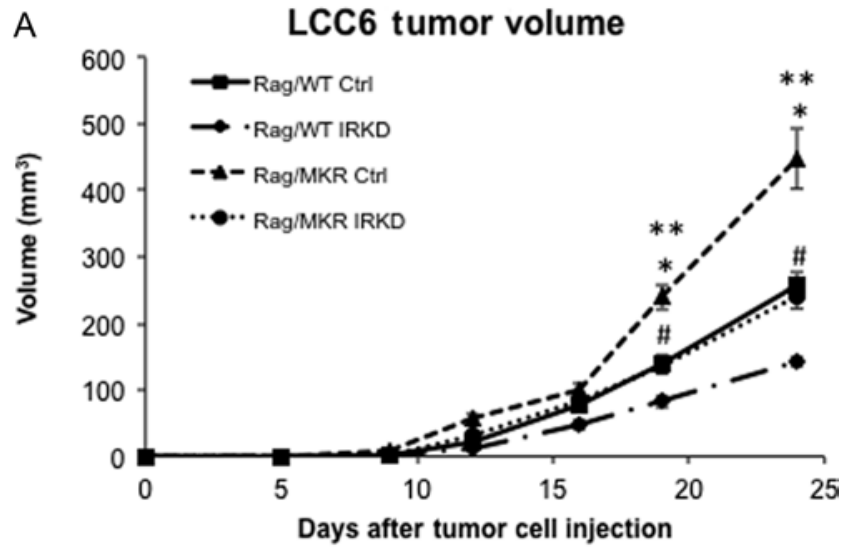

B LCC6 tumor weight

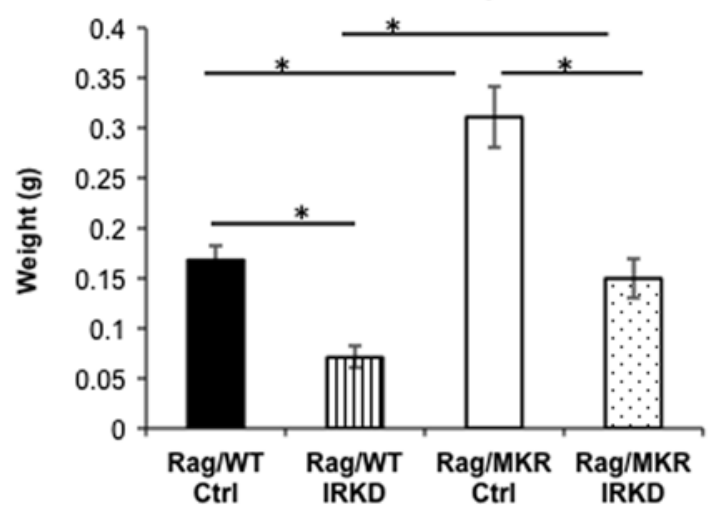

Figure 2

Silencing the insulin receptor leads to decreased tumor growth.

8-10-week-old Rag/WT control and Rag/MKR hyperinsulinemic mice were injected with either $5 \times 10^{6}$ LCC6 control (Ctrl) or $5 \times 10^{6}$ LCC6 insulin receptor knockdown (IRKD) cells, into the 4th mammary fat pad ( $n=8-10$ mice per group). (A) Mammary tumor volume was calculated from tumor measurements taken twice weekly with calipers. ${ }^{*} P<0.05$ between Rag/MKR Ctrl and Rag/WT Ctrl tumors. ${ }^{*} P<0.05$ between Rag/MKR Ctrl and Rag/MKR IRKD. $\# P<0.05$ between Rag/WT Ctrl and Rag/WT IRKD tumors. $n=8-10$ mice per group, error bars represent S.E.M. (B) Tumor weight at the end of the study ( ${ }^{*}<0.05$ between groups as indicated). $n=8-10$ mice per group, error bars represent S.E.M. 


\section{Cell culture}

The LCC6 parental, LCC6 control (Ctrl) and LCC6 insulin receptor knockdown (IRKD) clone IR6 cells are described previously (Zhang et al. 2010). Cells were grown in Dulbecco's Modified Eagle's Medium (DMEM) supplemented with $10 \%$ fetal bovine serum (FBS) (Invitrogen Life Technologies), $100 \mathrm{U} / \mathrm{mL}$ penicillin and $100 \mu \mathrm{g} / \mathrm{mL}$ streptomycin (Mediatech, Manassas, VA, USA). Cells transfected with shRNA were maintained in medium supplemented with $1.5 \mu \mathrm{g} / \mathrm{mL}$ puromycin. All cells were grown at $37^{\circ} \mathrm{C}$ in $5 \% \mathrm{CO} 2$ atmosphere.

\section{Western blotting}

LCC6 Ctrl and LCC6 IRKD cells and tumor tissue were lysed in ice-cold lysis buffer containing $50 \mathrm{mM}$ Tris, $150 \mathrm{mM} \mathrm{NaCl}, 1 \mathrm{mM}$ EDTA, $1.25 \%$ CHAPS, $1 \mathrm{mM}$ sodium orthovanadate, $10 \mathrm{mM}$ sodium pyrophosphate, $8 \mathrm{mM}$ $\beta$-glycerophosphate and Complete Protease Inhibitor Cocktail tablet (Roche). The protein concentration of the cells and tumors was measured using the BCA protein assay kit (Thermo Scientific). Twenty-five micrograms of protein sample were resuspended in $3 \times$ loading buffer supplemented with DTT (Cell Signaling Technologies). The samples were denatured at $96^{\circ} \mathrm{C}$ for $5 \mathrm{~min}$. The prepared samples were run on SDS-PAGE $8-16 \%$ Tris-glycine gel (Invitrogen Life Technologies) and transferred to nitrocellulose membrane. After overnight incubation at $4^{\circ} \mathrm{C}$ with primary antibodies, the membranes were incubated with secondary antibodies (Li-Cor Biosciences, Lincoln, NE, USA) and scanned using the Li-Cor infrared imaging system. The Western bands were quantified using open-source Image J software (National Institutes of Health, Bethesda, MD, USA).

\section{Antibodies}

The Western blotting nitrocellulose membranes were probed with the following primary antibodies: anti-phospho-IGF1 receptor $\quad \beta^{\text {(Tyr1150/1151)/-phospho }}$ IR $\beta^{\text {(Tyr1135/1136) }}$ anti-c-MYC, anti-ZEB1, anti-SLUG, anti-vimentin, anti-phospho-AKT(Ser473), anti-total AKT, anti-phospho p44/42 mitogen-activated protein

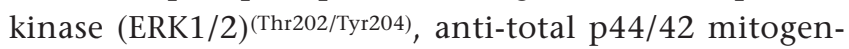
activated protein kinase (ERK1/2), anti-phospho S6 Ribosomal Protein(Ser235/236), anti-total S6 Ribosomal Protein (Cell Signaling Technology), anti-IR, antiIGF1R, anti-TWIST1 (Santa Cruz Biotechnology), and $\beta$-actin (Sigma-Aldrich).

\section{Metabolic studies}

Body weight was measured once a week from 3 to 10 weeks of age. Plasma in nonfasting state was collected in heparinized capillary tubes for insulin quantification. Plasma insulin levels were measured using the Ultra Sensitive Mouse Insulin ELISA kit according to manufacturer's instructions (Crystal Chem, Downers Grove, IL, USA). Serum adiponectin and serum leptin levels were measured using the Ultra Sensitive Mouse Adiponectin and Ultra Sensitive Leptin ELISA kits, respectively, according to manufacturer's instructions (Crystal Chem). Body composition was determined in nonanesthetized mice using the EchoMRI 3-in-1 NMR system (Echo Medical Systems, Houston, TX, USA). Insulin tolerance test was performed on 8-week-old
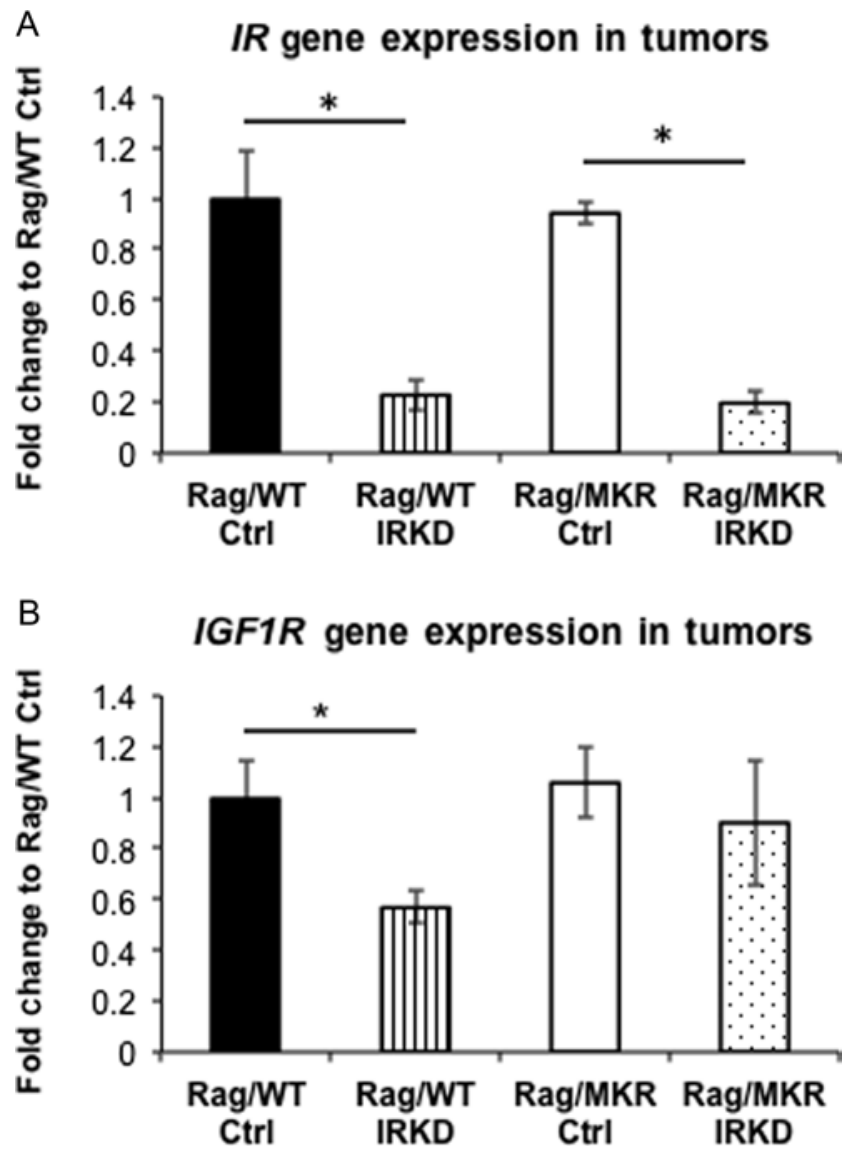

Figure 3

Expression of IR and IGF1R in LCC6 Tumors. (A) Primary tumors from Rag/ WT and Rag/MKR mice were assessed for the gene expression of the insulin receptor, demonstrating an $83 \%$ reduction of $I R$ in the tumors from the LCC6 IRKD cells. ( $P<0.05, n=8-10$ mice per group, error bars represent S.E.M.). (B) Primary tumors from Rag/WT and Rag/MKR mice were assessed for the gene expression of the insulin-like growth factor-1 receptor IGF1R ( ${ }^{*}<0.05 n=8-10$ mice per group, error bars are S.E.M.).

Published by Bioscientifica Ltd. 

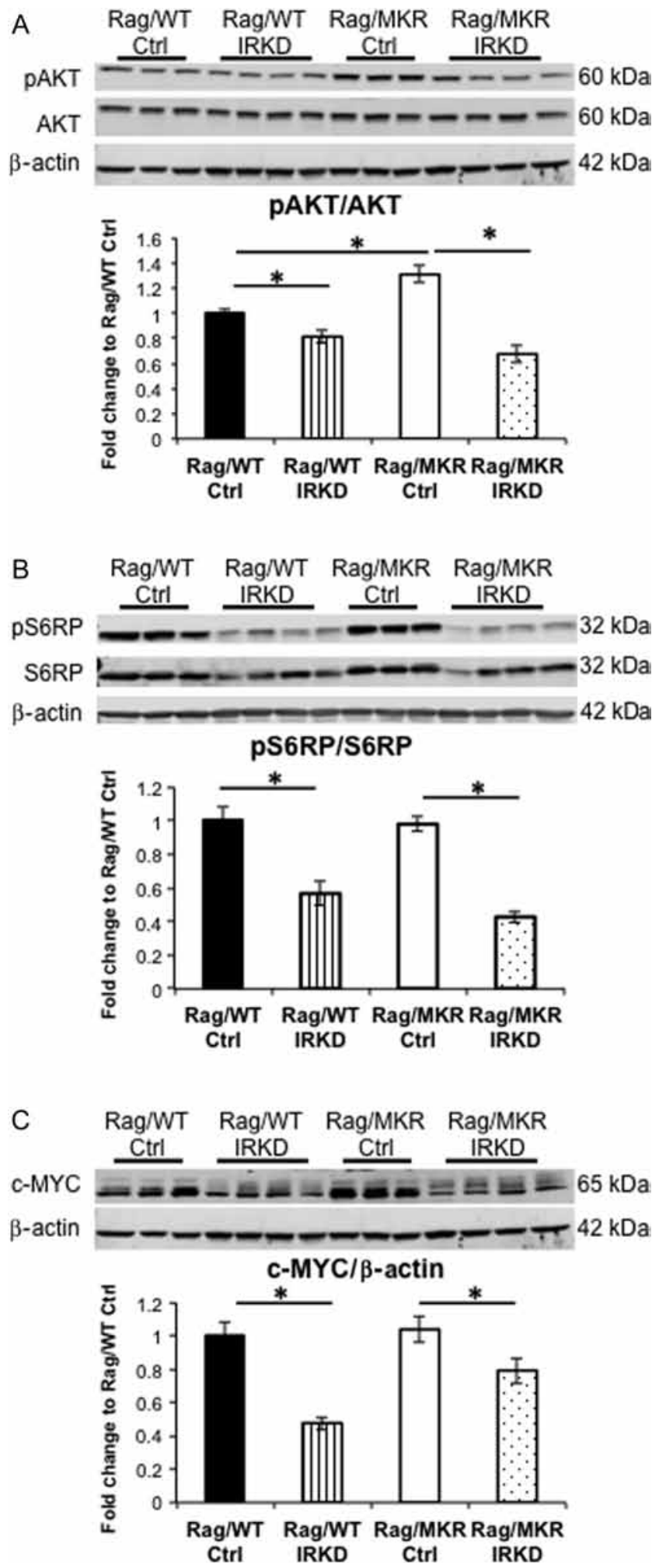

Figure 4

Reduction in insulin signaling pathway in LCC6 IRKD tumors. (A) Representative blots showing protein extracted from tumor tissue and analyzed by Western blotting for phospho-Akt (pAKT) and total AKT mice fasted for $2 \mathrm{~h}$. Insulin ( 0.75 units $/ \mathrm{kg}$ ) was injected intraperitoneally. Blood glucose levels were measured immediately before injection at time 0 and at 15, 30 and $60 \mathrm{~min}$ after insulin injection using Bayer Contour Next Glucometer (Bayer HealthCare, Mishawaka, IN, USA). Weekly blood glucose measurements on nonfasted animals were taken using Bayer Contour Next Glucometer (Bayer HealthCare). Glucose tolerance test was performed on 8 -week-old mice fasted for $8 \mathrm{~h}$. Glucose $(2 \mathrm{~g} / \mathrm{kg})$ was injected intraperitoneally. Blood glucose levels were measured immediately before glucose injection at time 0 and at 15, 30, 60 and 120 min using Bayer Contour Next Glucometer. Serum triglyceride and serum cholesterol levels were performed using the Pointe Scientific Liquid Triglyceride and Liquid Cholesterol kits, respectively, per the manufacturer's instructions (Pointe Scientific, Canton, MI, USA).

\section{Statistical analysis}

All data are expressed as mean \pm S.E.M. Student's $t$-test and, where appropriate, two-way ANOVA followed by Tukey HSD post hoc test, was used with $P$ value $\leq 0.05$ considered statistically significant using GraphPad Prism Software.

\section{Results}

\section{Metabolic phenotype of the Rag/MKR mice}

As the immune system may play a role in the development of insulin resistance (DeFuria et al. 2013), we characterized the metabolic phenotype of the $\operatorname{Rag}^{-/-} / \mathrm{MKR}^{+/+}$ (Rag/MKR) mice and Rag1-/- (Rag/WT) controls, in both male and female mice. Body weights were measured weekly. Male and female Rag/MKR mice demonstrated lower body weight, of approximately 3-4 g, compared with age- and gender-matched Rag/WT control mice throughout the from 3 to 10 weeks of age (Fig. 1A).

expression. $\beta$-actin antibody used as loading control. Densitometry of Western blotting ( ${ }^{*} P<0.05$, graphs represent mean per group $(n=8-10$ mice per group) and error bars represent S.E.M.). (B) Representative blots showing protein extracted from tumor tissue and analyzed by Western blotting for phospho-S6 ribosomal protein (pS6RP) and total S6RP expression. $\beta$-actin antibody used as loading control. Densitometry of Western blotting ( ${ }^{*} P<0.05$, graphs represent mean per group $(n=8-10$ mice per group) and error bars represent S.E.M.). (C) Representative blots showing protein extracted from tumor tissue and analyzed by Western blotting for C-MYC expression. $\beta$-actin antibody was used as the loading control. Densitometry of Western blotting of c-MYC/ $\beta$-actin $(* P<0.05$, graphs represent mean per group ( $n=8-10$ mice per group) and error bars are S.E.M.).
() 2016 Society for Endocrinology Printed in Great Britain 
In addition, they showed less whole body fat compared with Rag/WT controls at 9 weeks of age (Fig. 1B). Insulin tolerance tests were performed in all four groups and Rag/MKR male and female mice demonstrated severe insulin resistance, with no glucose reduction in response to the 0.75 Units/kg insulin injection over $60 \mathrm{~min}$, while in Rag/WT control mice blood glucose levels rapidly fell greater to less than $50 \%$ of baseline, $60 \mathrm{~min}$ after the insulin injection (Fig. 1C). The presence of endogenous hyperinsulinemia was confirmed by the measurement of nonfasting plasma insulin levels: $5.34 \mathrm{ng} / \mathrm{mL} \pm 0.45$ in 9-week-old female Rag/MKR mice compared with $1.01 \mathrm{ng} / \mathrm{mL} \pm 0.08$ in $\mathrm{Rag} / \mathrm{WT}$ female mice $(P<0.01)$ and $11.7 \mathrm{ng} / \mathrm{mL} \pm 3.55$ in 9-week-old Rag/MKR males compared with $1.69 \mathrm{ng} / \mathrm{mL} \pm 0.31 \mathrm{in} \mathrm{Rag} / \mathrm{WT}$ male mice (Fig. 1D). Male Rag/MKR mice presented with endogenous hyperglycemia, assessed by the measurement of nonfasting blood glucose levels (Supplementary Fig. 1A, see section on supplementary data given at the end of this article). Rag/MKR females present no signs of hyperglycemia, with nonfasting blood glucose levels comparable to Rag/WT male and female mice (Supplementary Fig. 1A). Furthermore, a glucose tolerance test was performed on 8-week-old mice after an $8 \mathrm{~h}$ fast (Supplementary Fig. 1B). The Rag/MKR males were hyperglycemic, while the Rag/MKR females demonstrated mild glucose intolerance compared with Rag/WT females, consistent with them having insulin resistance (Supplementary Fig. 1B). There was no difference in serum triglyceride or serum cholesterol levels between the Rag/WT and Rag/MKR mice (Supplementary Fig. 1C and D). There was no significant difference in serum adiponectin or serum leptin levels in the Rag/WT and the Rag/MKR mice (Supplementary Fig. 2A and B).

These results demonstrate that female MKR mice crossed with $\mathrm{Rag}^{-/-}$mice are not obese, not hyperglycemic and not dyslipidemic, but have insulin resistance with endogenous hyperinsulinemia and insulin levels that are approximately five times the control mice.

\section{The role of the insulin receptor in the effect of hyperinsulinemia on human cancer progression}

To examine the role of the insulin receptor (IR) in mediating the effect of the endogenous hyperinsulinemia on cancer progression, we studied the LCC6 IR6 clone cell line in which expression of the IR was markedly reduced by short hairpin RNA (Zhang et al. 2010) (Supplementary Fig. 3). Tumors derived from LCC6 Ctrl cells grew more rapidly in Rag/MKR mice compared with Rag/WT LCC6 Ctrl cells, measuring $447.3 \pm 45.0 \mathrm{~mm}^{3}$ and
$256.8 \pm 19.2 \mathrm{~mm}^{3}$, respectively, $P<0.05$ (Fig. $2 \mathrm{~A}$ ). Tumors derived from the LCC6 IRKD cells were significantly smaller than their control cell counterparts (Fig. 2A). Tumor weights were significantly greater in the Rag/MKR mice with Ctrl cells compared with Rag/WT mice with Ctrl cells 24 days after injection, weighing $0.31 \pm 0.03 \mathrm{~g}$ and $0.16 \pm 0.01 \mathrm{~g}$, respectively, $P<0.05$ (Fig. $2 \mathrm{~B}$ ). The tumors from the LCC6 IRKD cells in the Rag/MKR and Rag/WT mice weighed $0.15 \pm 0.02 \mathrm{~g}$ and $0.07 \pm 0.01 \mathrm{~g}$, respectively, $P<0.05$ (Fig. 2B). The reduction in tumor volume was seen in both Rag/WT and Rag/MKR mice. In both the Rag/WT mice and the Rag/MKR mice, the LCC6 IRKD tumor volume was decreased by half compared with respective Rag/WT and Rag/MKR with LCC6 Ctrl tumor volume.

\section{Examining the IR signaling pathway}

Sustained knockdown of the IR mRNA expression in the tumor xenografts was validated by qRT-PCR at the end of the study and an $83 \%$ reduction in $I R$ expression was confirmed (Fig. 3A). The IGF1R gene expression was decreased in the Rag/WT IRKD tumors, but there was no change in the IGF1R gene expression in the Rag/MKR Ctrl compared with the Rag/MKR IRKD tumors (Fig. 3B). These results confirm successful knockdown of the insulin receptor in the cells and in the tumors generated in the Rag/WT and Rag/MKR mice.
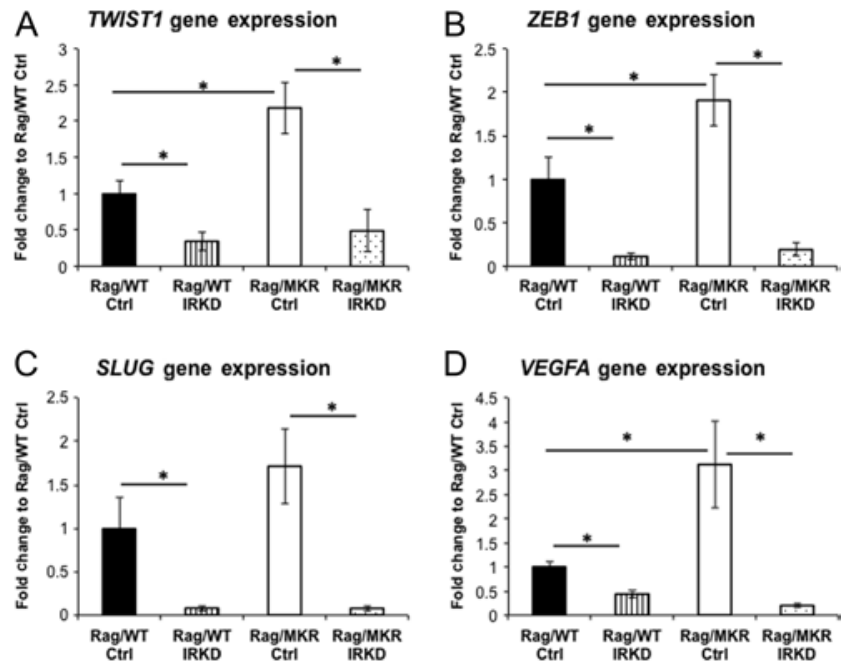

Figure 5

EMT and angiogenesis in Ctrl and IRKD tumors. RNA extracted from tumor tissue was analyzed for (A) TWIST1, (B) ZEB1, (C) SLUG and (D) Vascular endothelial growth factor A (VEGFA) gene expression by qRT-PCR. Calculations were made using ribosomal protein L19 (RPL19) as housekeeping gene. Graphs represent mean per group ( $n=5$ per group) and error bars are S.E.M. (* $P<0.05)$.

Published by Bioscientifica Ltd. 

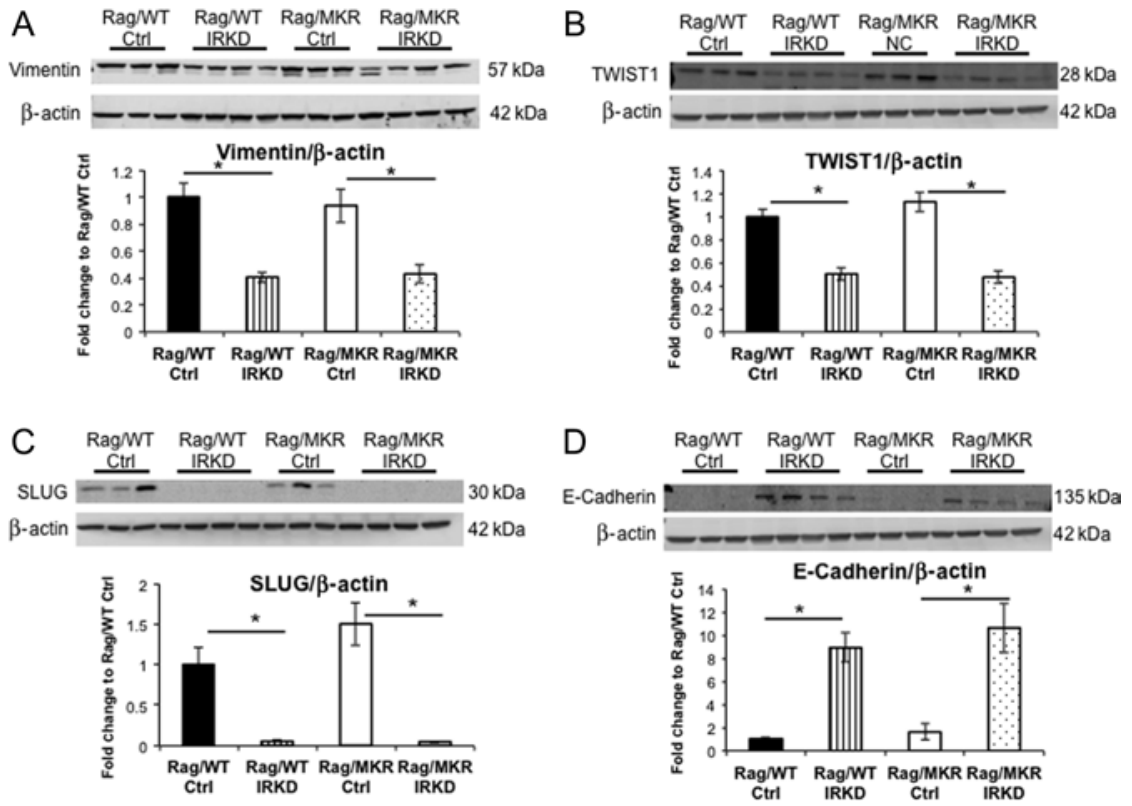

\section{Figure 6}

Tumors from LCC6 IRKD cells have reversal of epithelial-mesenchymal transition phenotype. (A) Representative blots showing protein extracted from tumor tissue and analyzed by Western blotting for vimentin expression. $\beta$-actin antibody was used as the loading control. Densitometry of Western blotting of vimentin/ $\beta$-actin. (B) Representative blots showing protein extracted from tumor tissue and analyzed by Western blotting for TWIST1 expression. $\beta$-actin antibody was used as the loading control. Densitometry of Western blotting TWIST1/ $\beta$-actin. (C) Representative blots showing protein extracted from tumor tissue and analyzed by Western blotting for SLUG expression. $\beta$-actin antibody used as loading control. Densitometry of Western blotting of SLUG/ $\beta$-actin. (D) Representative blots showing protein extracted from tumor tissue and analyzed by Western blotting for E-cadherin expression. $\beta$-actin antibody used as loading control. Densitometry of Western blotting of E-cadherin/ $\beta$-actin. Graphs represent mean per group ( $n=8-10$ mice) and error bars are S.E.M. $\star P<0.05$ between groups as indicated.
Examining the insulin receptor signaling pathway, a significant decrease in phospho-AKT (Ser473) was observed in the IRKD tumors from both the Rag/WT and Rag/MKR mice (Fig. 4A). Furthermore, an increase in phospho-AKT signaling was observed in the LCC6 Ctrl tumors from the Rag/MKR mice compared with the LCC6 Ctrl tumors from the Rag/WT mice (Fig. 4A). There was a decrease in the phospho-S6 Ribosomal Protein (S6RP) in the IRKD tumors from both the Rag/WT and Rag/MKR mice (Fig. 4B). Several studies have noted that insulin may have an effect on c-MYC expression (Mawson et al. 2005, Ferguson et al. 2012) and that the phosphatidylinositol 3-kinse (PI3K) and mammalian target of rapamycin (mTOR) signaling pathways may cooperate in regulating c-MYC expression (Sharma et al. 2015). Analyzing the proteins from the primary tumors, we found that the LCC6 IRKD tumors had decreased c-MYC protein levels compared with LCC6 Ctrl tumors (Fig. 4C). These results demonstrate that knockdown of the IR successfully decreases PI3K/AKT/ mTOR signaling pathway, downstream targets of IR signaling. Furthermore, the data show that reduction in IR signaling leads to a decrease in c-MYC protein levels.

\section{Reversal of EMT in IRKD tumors}

LCC6 Ctrl tumors in Rag/MKR mice had increased gene expression of TWIST1 and ZEB1 compared with Rag/WT Ctrl tumors (Fig. 5A and B). The increased SLUG
(SNAIL family transcription factor) gene expression in the LCC6 Ctrl tumors in the Rag/MKR mice compared with Rag/WT Ctrl tumors did not reach statistical significance (Fig. 5C). The primary tumors from the Rag/MKR LCC6 Ctrl mice also had significantly higher levels of VEGFA compared with the primary tumors from the Rag/WT LCC6 Ctrl mice (Fig. 5D). These results provide evidence that hyperinsulinemia promotes EMT and an increase in VEGFA, which may contribute to increased tumor growth and invasion in the Rag/MKR Ctrl tumors compared with the Rag/WT Ctrl tumors. Analyzing the LCC6 IRKD tumors, we found that gene expression of the mesenchymal transcription factors TWIST1, ZEB1 and SLUG was reduced in the LCC6 IRKD tumors in both the Rag/WT and Rag/MKR mice (Fig. 5A, $B$ and C). The LCC6 IRKD tumors also had decreased levels of angiogenesis markers VEGFA (Fig. 5D) and VEGFD (Supplementary Fig. 4). The primary tumors from both the Rag/WT and Rag/MKR LCC6 IRKD cells had decreased protein levels of mesenchymal markers vimentin, TWIST1 and SLUG compared with their LCC6 Ctrl tumors counterparts in the Rag/WT and Rag/MKR mice (Fig. 6A, B and C). Furthermore, the LCC6 IRKD primary tumors re-expressed E-cadherin, a classic epithelial marker (Fig. 6D). The knockdown of the IR promoted a reversal of the epithelialmesenchymal transition by repressing mesenchymal markers and re-expressing epithelial markers in the LCC6 IRKD tumors.

Published by Bioscientifica Ltd. 

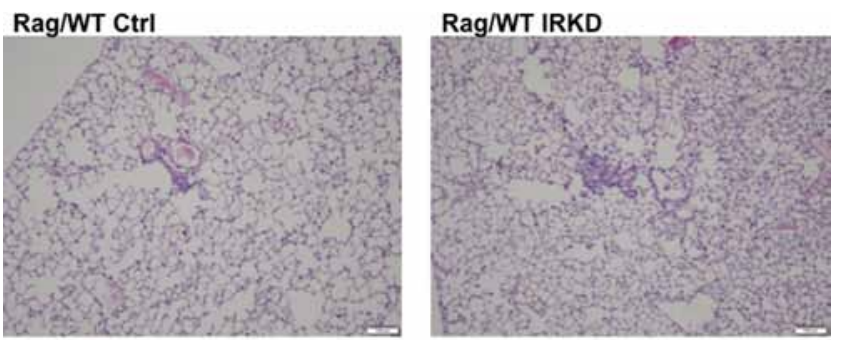

Rag/MKR Ctrl

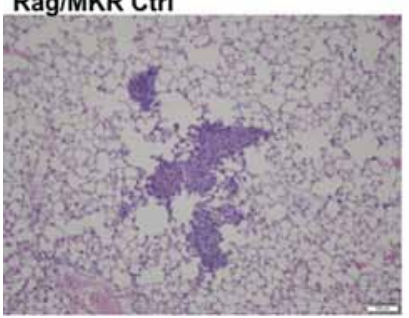

Rag/MKR IRKD

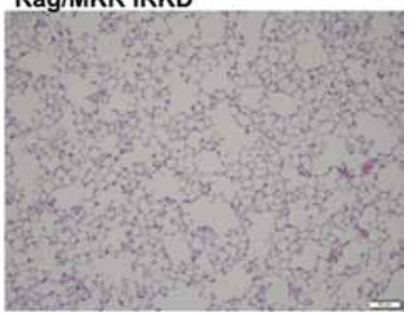

Figure 7

Insulin receptor knockdown inhibited pulmonary metastases in hyperinsulinemic mice. Lungs were fixed, paraffin embedded and sectioned. Representative images of H\&E-stained lung sections from Rag/WT and Rag/MKR mice injected with LCC6 Ctrl and LCC6 IRKD cells. (A and B) 0/5 (0\%) of Rag/WT injected with LCC6 Ctrl or LCC6 IRKD cells had pulmonary metastasis. (C) Representative pulmonary micrometastases seen in 2/5 (40\%) Rag/MKR injected with LCC6 control cells. (D) 0/5 (0\%) of Rag/MKR mice injected with LCC6 IRKD cells had pulmonary metastases. 10x magnification with scale bar of $100 \mu \mathrm{m}$ in length.

\section{IRKD clone had decreased number of lung metastases in hyperinsulinemic mice}

The LCC6 orthotopically injected cells have been shown to metastasize to the lungs. The lungs harvested 28 days post cancer cell inoculation were sectioned and examined for micrometastasis. In the Rag/WT mice, neither the mice with the LCC6 Ctrl tumors (0/5) nor the mice with the LCC6 IRKD tumors (0/5) had any pulmonary micrometastases. In the lungs from the hyperinsulinemic Rag/MKR group, 2/5 (40\%) of the mice with LCC6 Ctrl tumors had numerous lung micrometastases (Fig. 7), while 0/5 (0\%) lungs from the Rag/MKR mice with LCC6 IRKD tumors had micrometastasis. The data suggest that silencing the insulin receptor diminished cancer cell metastasis to the lung of the hyperinsulinemic mice, likely through the downregulation of mesenchymal proteins.

\section{Discussion}

Epidemiological studies have demonstrated the association between obesity and type 2 diabetes and an increased cancer risk and mortality of both epithelial such as colon, breast, endometrial cancers and nonsolid tumors, such as lymphomas (Haslam \& James 2005, Onitilo et al. 2012). Of the numerous potential factors that could explain this association, endogenous hyperinsulinemia has been identified as an important factor inversely correlating with survival in women with breast cancer (Goodwin et al. 2002, Eliassen et al. 2007). To determine whether endogenous hyperinsulinemia is a direct causal factor in promoting progression of human tumors, by acting on the tumor cell IR, we have generated a novel immunodeficient mouse model of endogenous hyperinsulinemia. The model is a female Rag/MKR mouse that (unlike the male mouse that shows the full phenotype of type 2 diabetes) exhibits severe insulin resistance and hyperinsulinemia, without hyperglycemia. This mouse thus represents the equivalent of the insulin resistance and endogenous hyperinsulinemia seen in obesity and prediabetes without the other confounding variables, such as dyslipidemia or the need for a high-fat diet. In fact, the Rag/MKR mice have lower body weight, lower fat mass, normal triglyceride, normal cholesterol and normal adiponectin levels compared with Rag/WT control mice. It would be expected that a decrease in fat mass would also lead to a decrease in leptin levels. In a study published previously by our group, we found that in MKR females there were lower leptin levels (Fierz et al. 2010a), while in MKR males there was no significant difference compared with control mice (Toyoshima et al. 2005). In this study, the Rag/MKR female mice had no significant difference in leptin levels compared with control mice, which may be due to the large error bars in the Rag/WT female group. This study demonstrated that we successfully generated an immunodeficient mouse model of both type 2 diabetes (male mice) and prediabetes (female mice). The difference between the degree of severity of diabetes between the male and female mice is likely attributed to estrogen in the female mice. In immunocompetent hyperinsulinemic MKR mice, ovariectomy led to the female MKR mice to develop additional symptoms of type 2 diabetes (hypercholesterolemia and hyperglycemia) (Ben-Shmuel et al. 2015). In other animal models, estrogen has been shown to reverse the adverse metabolic effects of ovariectomy (Zhu et al. 2013).

Similar to our previous studies, using mouse mammary tumors (Fernandez et al. 2001, Novosyadlyy et al. 2010, Ferguson et al. 2012), we have found that the Rag/MKR mice develop larger primary tumors with more numerous pulmonary metastasis when injected with human metastatic LCC6 cells (a variant of MDA-MB-435) that are highly malignant and metastasize (Price 1996, Holliday \& Speirs 2011). In our previous studies on murine cancers, we found that increased tumor promotion was reversed when the IR/IGF1Rs were blocked using a tyrosine kinase

Published by Bioscientifica Ltd. 
inhibitor or the hyperinsulinemia was pharmacologically reduced, thereby suggesting a role for insulin and the IR/ IGF1R in tumor progression (Novosyadlyy et al. 2010, Fierz et al. 2010a,b, Ferguson et al. 2012, Gallagher et al. 2012). However, in these studies, we could not examine the effects of hyperinsulinemia on human cancers.

It has previously been shown that constitutive activation of the IGF1R in nontransformed human mammary epithelial cells promoted increased proliferation, loss of contact inhibition, anchorageindependent growth, invasion and tumorigenesis (Kim et al. 2007). The MCF10A cells upregulated SNAIL mRNA levels (a mesenchymal transcription factor) and downregulated E-cadherin mRNA levels in response to IGF1R overexpression (Kim et al. 2007). Using a small molecule, inhibitor of IGF1R/IR reversed the EMT changes caused by the IGF1R overexpression in the MCF10A cells; an increase in E-cadherin mRNA levels was observed, as well as a decrease in the mesenchymal marker vimentin (Kim et al. 2007). The study by Kim and coworkers did not examine the expression of the IR in the setting of IGF1R overexpression. Our current study demonstrates that the female Rag/MKR mouse has more aggressive tumor growth compared with the Rag/WT mice. The data indicate that the IR expression on the tumor cells is important for tumor growth. However, in the Rag/MKR mice with the IRKD tumors, there was still an increase in tumor growth compared with the Rag/WT IRKD tumors. This may indicate that tumor growth may be mediated by the hyperinsulinemia in the Rag/MKR mice acting directly or indirectly, potentially by regulating IGFBP expression and local bioavailable IGF1 levels. The EMT changes seen in the Rag/MKR mice appear to be mediated directly by the effects of insulin on the insulin receptor. We have previously shown that in the MKR hyperinsulinemic mice, there is an upregulation of both the IR and IGF1R expression in the tumors compared with the tumors from control mice (Ferguson et al. 2012). The LCC6 IR knockdown tumors in the Rag/MKR mice have higher level of IGF1R than the LCC6 IR knockdown tumors from the Rag/WT mice. This could partially compensate for the IR knockdown in the Rag/MKR mice. Therefore, in the absence of the IR, hyperinsulinemia may promote tumor growth by acting directly or indirectly through the IGF1R.

The Rag/MKR LCC6 Ctrl primary tumors show elevated AKT/mTOR signaling, increased EMT gene expression of TWIST1 and ZEB1 and increased gene expression of VEGFA compared with the Rag/WT LCC6 Ctrl tumors. All of these factors may contribute to the increased tumor growth in the Rag/MKR mice. We have previously found an increase in phospho-AKT signaling in the primary tumors from MKR mice compared with controls and that inhibiting PI3K/AKT signaling reduces murine tumor growth (Novosyadlyy et al. 2010, Gallagher et al. 2012). Using the LCC6 IRKD cells, we demonstrated that the IRKD significantly reverses the effect of the hyperinsulinemia and that the tumors from the IRKD cells are significantly smaller in both the Rag/WT and Rag/MKR mice compared with the LCC6 Ctrl counterparts, and have less metastatic lesions. Moreover, we show that the Rag/MKR LCC6 Ctrl primary tumors have increased expression of VEGFA and VEGFD. VEGFA and $V E G F D$ are known mediator of angiogenesis that has been linked to tumor growth (Zhang et al. 2007, Calvo et al. 2008). In a work published previously from the lab, we found that there were increased levels of VEGF and in tumors from the MKR mice (Ferguson et al. 2012). The increased expression of VEGF may indicate increased angiogenesis, an important process in tumor growth and metastasis (Zhang et al. 2007, Calvo et al. 2008). The increased gene expression of VEGFA and VEGFD may contribute to the increased tumor size and numerous metastases in the hyperinsulinemic LCC6 Ctrl mice.

We show that IRKD leads to significant decreases in phospho-AKT, phospho-S6RP and in the expression of the cancer growth enhancing protein c-MYC. c-MYC is a transcription factor that is known to control cell cycle progression and differentiation ( $\mathrm{Xu}$ et al. 2010). Approximately, $50 \%$ of breast cancers are due to an oncogenic transformation, which leads to an overexpression of c-MYC through gene amplification, chromosomal transformation or protein overexpression events (Chrzan et al. 2001, Xu et al. 2010). It has been shown that using the dual PI3K/mTOR inhibitor, NVPBEZ235 inhibits the expression of c-MYC in pancreatic cancer cells (Sharma et al. 2015). Moreover, c-MYC has also been linked to promoting EMT induction in endometrial cancer cells (Liu et al. 2015). Therefore, the decrease in c-MYC protein expression in the IRKD tumors in both the Rag/WT and Rag/MKR mice may contribute to the significantly decreased tumor sizes and reversal of EMT in the tumors of those mice. Furthermore, the downregulation of the IR in the LCC6 cells significantly affected the formation of colonies in soft agar (Zhang et al. 2010). These results highlight the importance of the insulin receptor in proliferation, and the reversal of EMT may explain their inability to overcome anoikis.

The parent of the LCC6 cells, the MDA-MB-435 cell line, is highly metastatic and expresses many of the classic EMT markers such as low levels of E-cadherin and

Published by Bioscientifica Ltd. 
high levels of vimentin, N-cadherin and alpha smooth muscle actin (Manni et al. 2002, Li et al. 2014). In the current study, we show downregulated expression of mesenchymal markers including vimentin, TWIST1, SLUG (SNAIL family transcription factor) and ZEB1 at RNA and protein levels in the tumors with IR knockdown. While we saw an increase in TWIST1 and SLUG gene expression in the Rag/MKR Ctrl tumors compared with the Rag/WT Ctrl tumors, we did not see a significant difference in the protein levels of these genes between the Rag/MKR Ctrl and Rag/WT Ctrl tumors. This may be in part due to the short half-life of the TWIST and SNAIL transcription factor family proteins that are rapidly polyubiquitinated and degraded by the proteasome in cells (Diaz et al. 2014). These mesenchymal factors are essential markers of EMT, a biological process in which polarized epithelial cells undergo various changes to take on a mesenchymal phenotype. This phenotype includes increased migratory capacity, invasiveness, resistance to apoptosis and elevated production of extracellular matrix (ECM) components (Thiery 2002, Kalluri \& Weinberg 2009). In many carcinomas, growth factors such as transforming growth factor beta (TGFB) and epidermal growth factor (EGF) can lead to the induction of EMT signals by increasing the gene expression of various EMT-inducing transcription factors; most commonly, SNAIL, SLUG, zinc finger E-box binding homeobox 1 (ZEB1) and TWIST1 (Kim et al. 2002, Shi \& Massague 2003, Niessen et al. 2008, Spaderna et al. 2008, Al Moustafa et al. 2012, Pan et al. 2012). To our knowledge, the data presented in this study are the first to show that hyperinsulinemia increases the expression of several EMT genes in vivo. ZEB1 has been shown to induce EMT and downregulate E-cadherin in epithelial cells (Singh et al. 2008). Moreover, ZEB1 knockdown in nonsmall cell lung cancer cell lines suppressed the growth and colony formation of the cells (Takeyama et al. 2010). In the same study, they noted that ZEB1 knockdown induced E-cadherin mRNA expression in some of the nonsmall cell lung cancer cell lines (Takeyama et al. 2010). It has been shown that re-expression of E-cadherin can partially revert mesenchymal breast cancer cells toward an epithelial phenotype, as well as suppress migration and invasion of the cells (Chao et al. 2010).

In data published previously, it has been shown that the parent LCC6 cells readily metastasize in athymic mice (Zhang et al. 2010). In this study, we show that the LCC6 Ctrl cells did not metastasize in the Rag/WT mice, but did metastasize in the Rag/MKR mice. The discrepancy between the previous published data and the lack of metastases in the Rag/WT mice in the current study may relate to either differences in the mouse strains or the time to assess the number of pulmonary metastases. However, it is important to note that the LCC6 Ctrl primary tumors of the Rag/MKR mice showed increased levels of phospho-AKT and higher gene expression of TWIST1, ZEB1 and VEGFA compared with the LCC6 Ctrl tumors of Rag/WT mice, which can contribute to the increased tumor growth and metastases seen in the Rag/MKR LCC6 Ctrl tumors. The data presented in this study also show that insulin receptor knockdown leads to a decrease in ZEB1 transcriptional activity, which promotes the re-expression of E-cadherin, which was not previously showed by Zhang and coworkers. This may lead to decreased colony formation and decreased number of pulmonary metastasis formation.

In summary, we demonstrate that the female Rag/MKR mouse model is a novel immunodeficient model for prediabetes. This mouse model can be used to study many other human tumor types where hyperinsulinemia may play a role. The current study showed that silencing the insulin receptor in the LCC6 tumor cells decreased the tumor growth in both the Rag/WT and Rag/MKR mice. We show for the first time that hyperinsulinemic mice have increased EMT gene expression, increased VEGFA expression and have more pulmonary metastases. We also show for the first time that silencing the insulin receptor leads to a reversal of the EMT phenotype where these tumors were able to re-express E-cadherin and downregulate vimentin, TWIST1, SLUG and ZEB1 expression. Furthermore, the tumors from the insulin receptor knockdown cells had significantly decreased phospho-AKT and c-MYC expression, even in the setting of hyperinsulinemia. This suggests that reducing insulin receptor signaling or expression specifically in the tumors may lead to reduced number of pulmonary metastases.

\section{Supplementary data}

This is linked to the online version of the paper at http://dx.doi.org/10.1530/ ERC-16-0142.

\section{Declaration of interest}

The authors declare that there is no conflict of interest that could be perceived as prejudicing the impartiality of the research reported.

\section{Funding}

This work was supported by the NIH/NCl grant (2R01CA128799-06A1) and American Diabetes Association grant (1-13-BS-108) to Dr Derek LeRoith and the $\mathrm{NIH} / \mathrm{NCl}$ grant 1K08CA190770 to Dr Emily Jane Gallagher.

Published by Bioscientifica Ltd. 


\section{References}

Al Moustafa AE, Achkhar A \& Yasmeen A 2012 EGF-receptor signaling and epithelial-mesenchymal transition in human carcinomas. Frontiers in Bioscience 4 671-684. (doi:10.2741/s292)

Alikhani N, Ferguson RD, Novosyadlyy R, Gallagher EJ, Scheinman EJ, Yakar S \& LeRoith D 2013 Mammary tumor growth and pulmonary metastasis are enhanced in a hyperlipidemic mouse model. Oncogene 32 961-967. (doi:10.1038/onc.2012.113)

Ben-Shmuel S, Scheinman EJ, Rashed R, Orr ZS, Gallagher EJ, LeRoith D \& Rostoker R 2015 Ovariectomy is associated with metabolic impairments and enhanced mammary tumor growth in MKR mice. Journal of Endocrinology 227 143-151. (doi:10.1530/JOE-15-0310)

Calvo A, Catena R, Noble MS, Carbott D, Gil-Bazo I, GonzalezMoreno O, Huh JI, Sharp R, Qiu TH, Anver MR, et al. 2008 Identification of VEGF-regulated genes associated with increased lung metastatic potential: functional involvement of tenascin-C in tumor growth and lung metastasis. Oncogene 27 5373-5384. (doi:10.1038/onc.2008.155)

Chambers AF 2009 MDA-MB-435 and M14 cell lines: identical but not M14 melanoma? Cancer Research 69 5292-5293. (doi:10.1158/00085472.CAN-09-1528)

Chao YL, Shepard CR \& Wells A 2010 Breast carcinoma cells re-express E-cadherin during mesenchymal to epithelial reverting transition. Molecular Cancer 9 179. (doi:10.1186/1476-4598-9-179)

Chrzan P, Skokowski J, Karmolinski A \& Pawelczyk T 2001 Amplification of c-myc gene and overexpression of c-Myc protein in breast cancer and adjacent non-neoplastic tissue. Clinical Biochemistry 34 557-562. (doi:10.1016/S0009-9120(01)00260-0)

DeFuria J, Belkina AC, Jagannathan-Bogdan M, Snyder-Cappione J, Carr JD, Nersesova YR, Markham D, Strissel KJ, Watkins AA, Zhu M, et al. 2013 B cells promote inflammation in obesity and type 2 diabetes through regulation of T-cell function and an inflammatory cytokine profile. PNAS 110 5133-5138. (doi:10.1073/ pnas.1215840110)

Diaz VM, Vinas-Castells R \& Garcia de Herreros A 2014 Regulation of the protein stability of EMT transcription factors. Cell Adhesion \& Migration 8 418-428. (doi:10.4161/19336918.2014.969998)

Eliassen AH, Tworoger SS, Mantzoros CS, Pollak MN \& Hankinson SE 2007 Circulating insulin and c-peptide levels and risk of breast cancer among predominately premenopausal women. Cancer Epidemiology, Biomarkers \& Prevention 16 161-164. (doi:10.1158/ 1055-9965.EPI-06-0693)

Ferguson RD, Novosyadlyy R, Fierz Y, Alikhani N, Sun H, Yakar S \& Leroith D 2012 Hyperinsulinemia enhances c-Myc-mediated mammary tumor development and advances metastatic progression to the lung in a mouse model of type 2 diabetes. Breast Cancer Research 14 R8. (doi:10.1186/bcr3089)

Fernandez AM, Kim JK, Yakar S, Dupont J, Hernandez-Sanchez C, Castle AL, Filmore J, Shulman GI \& Le Roith D 2001 Functional inactivation of the IGF-I and insulin receptors in skeletal muscle causes type 2 diabetes. Genes \& Development 15 1926-1934. (doi:10.1101/gad.908001)

Fierz Y, Novosyadlyy R, Vijayakumar A, Yakar S \& LeRoith D 2010a Insulin-sensitizing therapy attenuates type 2 diabetes-mediated mammary tumor progression. Diabetes 59 686-693. (doi:10.2337/ db09-1291)

Fierz Y, Novosyadlyy R, Vijayakumar A, Yakar S \& LeRoith D $2010 b$ Mammalian target of rapamycin inhibition abrogates insulin-mediated mammary tumor progression in type 2 diabetes. Endocrine-Related Cancer 17 941-951. (doi:10.1677/ erc-10-0091)

Gallagher EJ, Fierz Y, Vijayakumar A, Haddad N, Yakar S \& LeRoith D 2012 Inhibiting PI3K reduces mammary tumor growth and induces hyperglycemia in a mouse model of insulin resistance and hyperinsulinemia. Oncogene 31 3213-3222. (doi:10.1038/ onc.2011.495)

Goodwin PJ, Ennis M, Pritchard KI, Trudeau ME, Koo J, Madarnas Y, Hartwick W, Hoffman B \& Hood N 2002 Fasting insulin and outcome in early-stage breast cancer: results of a prospective cohort study. Journal of Clinical Oncology 20 42-51. (doi:10.1200/ JCO.20.1.42)

Haslam DW \& James WP 2005 Obesity. Lancet 366 1197-1209. (doi:10.1016/S0140-6736(05)67483-1)

Holliday DL \& Speirs V 2011 Choosing the right cell line for breast cancer research. Breast Cancer Research 13 215. (doi:10.1186/bcr2889)

Howe LR, Subbaramaiah K, Hudis CA \& Dannenberg AJ 2013 Molecular pathways: adipose inflammation as a mediator of obesity-associated cancer. Clinical Cancer Research 19 6074-6083. (doi:10.1158/ 1078-0432.CCR-12-2603)

Hursting SD, Digiovanni J, Dannenberg AJ, Azrad M, Leroith D, Demark-Wahnefried W, Kakarala M, Brodie A \& Berger NA 2012 Obesity, energy balance, and cancer: new opportunities for prevention. Cancer Prevention Research 5 1260-1272. (doi:10.1158/1940-6207.CAPR-12-0140)

Kalluri R \& Weinberg RA 2009 The basics of epithelial-mesenchymal transition. Journal of Clinical Investigation 119 1420-1428. (doi:10.1172/JCI39104)

Kim K, Lu Z \& Hay ED 2002 Direct evidence for a role of beta-catenin/ LEF-1 signaling pathway in induction of EMT. Cell Biology International 26 463-476. (doi:10.1006/cbir.2002.0901)

Kim HJ, Litzenburger BC, Cui X, Delgado DA, Grabiner BC, Lin X, Lewis MT, Gottardis MM, Wong TW, Attar RM, et al. 2007 Constitutively active type I insulin-like growth factor receptor causes transformation and xenograft growth of immortalized mammary epithelial cells and is accompanied by an epithelial-to-mesenchymal transition mediated by NF-kappaB and snail. Molecular and Cellular Biology 27 3165-3175. (doi:10.1128/MCB.01315-06)

Li J, Liu J, Li P, Mao X, Li W, Yang J \& Liu P 2014 Loss of LKB1 disrupts breast epithelial cell polarity and promotes breast cancer metastasis and invasion. Journal of Experimental and Clinical Cancer Research 33 70. (doi:10.1186/s13046-014-0070-0)

Liu L, Zhang J, Yang X, Fang C, Xu H \& Xi X 2015 SALL4 as an epithelial-mesenchymal transition and drug resistance inducer through the regulation of c-Myc in endometrial cancer. PLOS ONE $\mathbf{1 0}$ e0138515. (doi:10.1371/journal.pone.0138515)

Manni A, Washington S, Griffith JW, Verderame MF, Mauger D, Demers LM, Samant RS \& Welch DR 2002 Influence of polyamines on in vitro and in vivo features of aggressive and metastatic behavior by human breast cancer cells. Clinical and Experimental Metastasis 19 95-105. (doi:10.1023/A:1014536909007)

Mawson A, Lai A, Carroll JS, Sergio CM, Mitchell CJ \& Sarcevic B 2005 Estrogen and insulin/IGF-1 cooperatively stimulate cell cycle progression in MCF-7 breast cancer cells through differential regulation of c-Myc and cyclin D1. Molecular and Cellular Endocrinology 229 161-173. (doi:10.1016/j.mce.2004.08.002)

Mombaerts P, Iacomini J, Johnson RS, Herrup K, Tonegawa S \& Papaioannou VE 1992 RAG-1-deficient mice have no mature B and T lymphocytes. Cell 68 869-877. (doi:10.1016/0092-8674(92)90030-G)

Morris DL, Cho KW, Delproposto JL, Oatmen KE, Geletka LM, MartinezSantibanez G, Singer K \& Lumeng CN 2013 Adipose tissue macrophages function as antigen-presenting cells and regulate adipose tissue CD4+ T cells in mice. Diabetes 62 2762-2772. (doi:10.2337/db12-1404)

Niessen K, Fu Y, Chang L, Hoodless PA, McFadden D \& Karsan A 2008 Slug is a direct Notch target required for initiation of cardiac cushion cellularization. Journal of Cell Biology 182 315-325. (doi:10.1083/jcb.200710067)

Novosyadlyy R, Lann DE, Vijayakumar A, Rowzee A, Lazzarino DA, Fierz Y, Carboni JM, Gottardis MM, Pennisi PA, Molinolo AA, et al. 2010 Insulin-mediated acceleration of breast cancer development http://erc.endocrinology-journals.org DOI: 10.1530/ERC-16-0142
๑ 2016 Society for Endocrinology Printed in Great Britain 
and progression in a nonobese model of type 2 diabetes. Cancer Research 70 741-751. (doi:10.1158/0008-5472.CAN-09-2141)

Onitilo AA, Engel JM, Glurich I, Stankowski RV, Williams GM \& Doi SA 2012 Diabetes and cancer I: risk, survival, and implications for screening. Cancer Causes and Control 23 967-981. (doi:10.1007/ s10552-012-9972-3)

Pan B, Ren H, He Y, Lv X, Ma Y, Li J, Huang L, Yu B, Kong J, Niu C, et al. $2012 \mathrm{HDL}$ of patients with type 2 diabetes mellitus elevates the capability of promoting breast cancer metastasis. Clinical Cancer Research 18 1246-1256. (doi:10.1158/1078-0432.CCR-11-0817)

Price JE 1996 Metastasis from human breast cancer cell lines. Breast Cancer Research and Treatment 39 93-102. (doi:10.1007/BF01806081)

Sharma N, Nanta R, Sharma J, Gunewardena S, Singh KP, Shankar S \& Srivastava RK 2015 PI3K/AKT/mTOR and sonic hedgehog pathways cooperate together to inhibit human pancreatic cancer stem cell characteristics and tumor growth. Oncotarget 6 32039-32060. (doi:10.18632/oncotarget.5055)

Shi Y \& Massague J 2003 Mechanisms of TGF-beta signaling from cell membrane to the nucleus. Cell 113 685-700. (doi:10.1016/S00928674(03)00432-X)

Singh M, Spoelstra NS, Jean A, Howe E, Torkko KC, Clark HR, Darling DS, Shroyer KR, Horwitz KB, Broaddus RR, et al. 2008 ZEB1 expression in type I vs type II endometrial cancers: a marker of aggressive disease. Modern Pathology 21 912-923. (doi:10.1038/ modpathol.2008.82)

Spaderna S, Schmalhofer O, Wahlbuhl M, Dimmler A, Bauer K, Sultan A, Hlubek F, Jung A, Strand D, Eger A, et al. 2008 The transcriptional repressor ZEB1 promotes metastasis and loss of cell polarity in cancer. Cancer Research 68 537-544. (doi:10.1158/0008-5472.CAN-07-5682)
Takeyama Y, Sato M, Horio M, Hase T, Yoshida K, Yokoyama T, Nakashima H, Hashimoto N, Sekido Y, Gazdar AF, et al. 2010 Knockdown of ZEB1, a master epithelial-to-mesenchymal transition (EMT) gene, suppresses anchorage-independent cell growth of lung cancer cells. Cancer Letters 296 216-224. (doi:10.1016/j. canlet.2010.04.008)

Thiery JP 2002 Epithelial-mesenchymal transitions in tumour progression. Nature Reviews Cancer 2 442-454. (doi:10.1038/nrc822)

Toyoshima Y, Gavrilova O, Yakar S, Jou W, Pack S, Asghar Z, Wheeler MB \& LeRoith D 2005 Leptin improves insulin resistance and hyperglycemia in a mouse model of type 2 diabetes. Endocrinology 146 4024-4035. (doi:10.1210/en.2005-0087)

$\mathrm{Xu}$ J, Chen Y \& Olopade OI 2010 MYC and breast cancer. Genes \& Cancer 1 629-640. (doi:10.1177/1947601910378691)

Zhang J, Lu A, Beech D, Jiang B \& Lu Y 2007 Suppression of breast cancer metastasis through the inhibition of VEGF-mediated tumor angiogenesis. Cancer Therapy 5 273-286.

Zhang H, Fagan DH, Zeng X, Freeman KT, Sachdev D \& Yee D 2010 Inhibition of cancer cell proliferation and metastasis by insulin receptor downregulation. Oncogene 29 2517-2527. (doi:10.1038/ onc.2010.17)

Zhang PH, Chen ZW, Lv D, Xu YY, Gu WL, Zhang XH, Le YL, Zhu HH \& Zhu YM 2012 Increased risk of cancer in patients with type 2 diabetes mellitus: a retrospective cohort study in China. BMC Public Health 12 567. (doi:10.1186/1471-2458-12-567)

Zhu L, Brown WC, Cai Q, Krust A, Chambon P, McGuinness OP \& Stafford JM 2013 Estrogen treatment after ovariectomy protects against fatty liver and may improve pathway-selective insulin resistance. Diabetes 62 424-434. (doi:10.2337/db11-1718)

Received in final form 2 July 2016

Accepted 19 July 2016

Accepted Preprint published online 19 July 2016
() 2016 Society for Endocrinology Printed in Great Britain
Published by Bioscientifica Ltd. 